\title{
EL OFICIO DEL FILÓSOFO Y LA TÉCNICA
}

\author{
Maximiliano Prada Dussán* \\ Universidad Nacional de Colombia. ${ }^{1}$
}

\section{Resumen}

Este artículo se pregunta por el oficio del filósofo en relación con la técnica; recorre los planteamientos de Bacon con respecto a la noción de utilidad del saber y el papel de la técnica. A continuación se realiza una exploración sobre la función de las telecomunicaciones y del entorno que ellas crean, en relación con la enseñanza, la investigación y el quehacer de la filosofía. Se exploran algunas de las direcciones de las tecnologías actuales de la información, señalando cómo el conocimiento se va desprendiendo de la naturaleza en busca de esa dirección

Palabras clave: Bacon, técnica, filosofía, informática.

\section{Abstract}

\section{The craft and the technique of the philosopher.}

The question posed in this essay is the relationship between the craft and technique of the philosopher. In this context, Bacon's philosophy is used in relation to the notion of utility of knowledge and the role of technique. Subsequently, we develop an exploration of the role of telecommunications, as well as the environment in which they are created, in regards to teaching, research and the task of philosophy. Moreover, this essay explores some of the directions of current information technologies, pointing to how knowledge is separated from its nature when it goes in this direction.

Key Words: Bacon, technique, computer technology.

\section{INTRODUCCIÓN}

Como el título lo indica, el asunto de este escrito es la pregunta por el oficio del filósofo en relación con la técnica; pregunta que, a su vez, se enmarca dentro de un interés particular que indaga por la formación en filosofía. En efecto, formar no es sólo cuestión de doctrinas, teorías y corrientes; tampoco es sólo cuestión de la apropiación de discursos y lenguajes

\footnotetext{
*aprada@pedagogica.edu.co

${ }^{1}$ Las ideas aquí recogidas son fruto de las investigaciones del último año dentro del grupo Filosofía y enseñanza de la filosofía, y dentro de algunos seminarios de la Maestría en Filosofía de la Universidad Nacional con el profesor Gonzalo Serrano.
} 
propios de la disciplina; es también asumir actitudes, ejercer prácticas, construir un rol; es configurar un oficio propio: el oficio del filósofo.

Un ejemplo familiar a nuestras universidades basta para ilustrar lo anterior. Se trata de las lecciones de filosofía dictadas por Manuel García Morente en la Universidad Nacional de Tucumán, que luego fueron recogidas en un texto llamado Lecciones preliminares de filosofía. En ellas, la filosofía y su enseñanza se presentan no como un cuerpo de doctrinas, sino como un hacer, recordando la distinción kantiana entre aprender filosofía y aprender a filosofar. Un acercamiento a ese hacer, a filosofar, es el tema de este escrito. No obstante, por considerar que este es un campo que está por construirse, no intentaremos dar cuenta totalmente de lo que implica filosofar; solamente queremos señalar unas líneas por las cuales se guía esta pregunta hoy, en medio de una sociedad tecnologizada, tomando por referencia aspectos como la actitud y las prácticas filosóficas.

La actitud y las prácticas filosóficas pueden variar según los tiempos, contextos y escuelas. Por ejemplo, de la ampliamente conocida actitud de asombro de los presocráticos, pasamos a la duda cartesiana, luego a la crítica y a la sospecha en filósofos contemporáneos; cada uno de ellos respondiendo a necesidades de sus momentos particulares -asunto que no vale la pena ahondar por el momento-. Por eso, es necesario situar la pregunta en un contexto concreto: hemos elegido situarnos en la configuración social y cultural que está sustentada en las teletecnologías, esto es, en lo que Javier Echeverría ha denominado Tercer Entorno, como aquel entorno social, de interacción humana, posibilitado por dichas tecnologías. Situarla en este contexto no es un asunto superficial; en lugar de ello, con esto se afirma que la pregunta por el filósofo en relación con la técnica debe ser replanteada a la luz de la aparición e impacto de las tecnologías de la información y la comunicación, que deben estudiarse las nuevas condiciones para la enseñanza, la investigación y la reflexión filosófica que configuran estas nuevas tecnologías y que nos es dado sospechar de si las reflexiones que generó la modernidad sobre estos asuntos siguen siendo hoy piezas relevantes capaces de guiar nuestra relación con la técnica.

Cabe destacar que este asunto deja por fuera, por el momento, muchos de los interrogantes que plantea la filosofía de la tecnología. No es mi interés ahora indagar en algunas de las preguntas que han lanzado los filósofos de la técnica en cuanto a su esencia o poder que ellas tienen; esto será abordado sólo en la medida en que pueda contribuir a la pregunta central esbozada más arriba.

Sin duda alguna, desde esta perspectiva se hace interesante el estudio de Francis Bacon, pues gran parte de su obra consistió en marcar pautas para el trabajo del filósofo en una era que estaba descubriendo el poder de la técnica. Más aún, podríamos aventurarnos a decir que es uno de los filósofos que más ha trabajado este campo de estudio. No obstante, al estudiar a este autor debemos enfrentar lo que la tradición ha dicho sobre él, en concreto, la acusación que se le ha hecho de utilitarista, imagen que surge sobre todo de una lectura positivista del filósofo inglés. Es importante tratar este asunto, pues al desligar la técnica del ethos utilitarista, se abren nuevas posibilidades frente a ella. Aquí proponemos una lectura de Bacon que deja a un lado esta acusación y que de alguna manera logra indicar elementos centrales que delimitan el oficio del filósofo frente a la técnica. Su estudio nos 
dará una primera idea de los aspectos que deben considerarse dentro de lo que nos proponemos. Este será el asunto en la primera parte del escrito.

La alusión a Bacon, además de ayudar a configurar el campo propio de nuestra pregunta, permitirá caracterizar la idea del filósofo y de la técnica dentro de un paradigma técnico específico: cuando la técnica se entiende como máquina. Atendiendo a los actuales cambios de paradigma tecnológico, marcados por la aparición de las tecnologías de la información y la comunicación, y que encontraremos esbozados en la obra de Ortega, es lícito pensar que debe rehacerse una reflexión sobre el filósofo a la luz de estos cambios. Para ello, desde luego, es necesario caracterizar lo que hoy comprendemos por tecnología, comprender por qué podemos hablar de un cambio tal que nos hace redefinir nuestros patrones de acción. A esto dedicaremos la segunda parte.

Arriesgaremos, en el tercer apartado, algunas líneas de trabajo que hoy exige la tecnología al filósofo. Para ello tomaremos un camino que, a partir de la analogía con lo esbozado sobre Bacon, nos permite plantear cuestiones presentes. Por último, haremos algunas consideraciones finales acerca de las posibilidades y los límites de la propuesta de trabajo con tecnología.

\section{Bacon, la técnica y el filósofo}

Como señala Pérez Ramos en Francis Bacon's Idea of Science: and the Maker's Knoledge Tradition, un posible punto de partida para interpretar la filosofía de Bacon es aquel que se refiere a la valoración del saber operativo por encima del contemplativo ${ }^{2}$. Este asunto permite tener una doble perspectiva sobre el autor: lo encontramos, por un lado, como un fuerte opositor de la manera tradicional de entender el saber, esto es, destruyendo la idea de saber proveniente de la escolástica y del renacimiento y, por otro, como uno de los pilares o antecedentes fundamentales de un punto de vista tecnológico sobre el conocimiento, desarrollado fundamentalmente en el siglo XX. Esta doble perspectiva intenta destacar, por un lado, el quiebre en la racionalidad imperante efectuado por Bacon; por otro, intentaría ahondar en una interpretación distinta del autor a partir de los problemas discutidos en la filosofía de la tecnología actual.

Antonio Pérez Ramos enfrenta la acusación corriente que se hace a Bacon según la cual el intento de fusionar el saber teórico con el práctico, en la idea de un saber operativo, es ideológico, en tanto es potencialmente una expresión del utilitarismo. En efecto, usualmente el proyecto baconiano es entendido como una mera producción de artefactos, y su noción de ciencia, como un saber que quiere imponerse al conocimiento filosófico disputativo y que desdibuja el papel propio de la filosofía de liberar al hombre del mundo material $^{3}$. Bacon es visto, también, sobrepasando los límites de un mero desarrollo técnico bajo el intento de extender "el saber técnico a los campos político, económico y moral", por ejemplo en la idea de una Nueva Atlántida, de modo que en estos ámbitos la acción de los

\footnotetext{
${ }^{2}$ Antonio Pérez-Ramos. Francis Bacon's idea of science: and the Maker's Knowledge Tradition. Oxford: Clarendon Press, 1988, p. 135.

${ }^{3}$ Ibídem, pp. 136-137.
} 
hombres sea también una acción técnica ${ }^{4}$; y es corriente encontrar que se le recuerda por su afán de dominio del hombre sobre la naturaleza.

Pérez Ramos circunscribe el proyecto baconiano en el campo epistemológico y lo libera del intento de emparentarlo con un proyecto ideológico que busque el dominio por sí mismo en el terreno ético y social, es decir, de considerar la técnica como el ethos de una cultura tecnológicamente orientada, al considerar que es distinto entender la utilidad en uno y otro campo ${ }^{5}$. El primero haría referencia a lo que Max Weber entiende por utilidad, esto es, un tipo de racionalidad que hace un balance entre medios y fines; mientras el segundo, a la idea de utilidad como sistema ético. El caso de Bacon, como se indicó, es el primero, toda vez que su proyecto es el de reformular el conocimiento de la naturaleza desde el punto de vista de investigar "tanto la acción a desempeñar como el producto de la acción" (Works, Opera $)^{6}$, de modo que la ciencia sea provechosa para el hombre. Dentro de este proyecto, la preocupación de la ciencia, como opera (Work), no es simplemente la producción de objetos, herramientas o artefactos para el provecho humano; la ciencia debe penetrar en las leyes mismas de la acción de la naturaleza.

Por esto, Pérez Ramos ve en el filósofo inglés no un germen del utilitarismo, sino una epistemología alternativa a la imperante en el renacimiento, en tanto entiende el conocimiento de la naturaleza no como el descubrimiento de esencias dentro del esquema clasificatorio de los seres heredado de la escolástica, sino como el descubrimiento de las acciones, operaciones o leyes por las cuales un fenómeno es producido, a lo cual denomina formas: "así, la forma del calor o la forma de la luz es absolutamente la misma cosa que la ley del calor o la ley de la luz"7. El conocimiento de las formas tiene tanto que ver con la verdad como con la aplicación: "Pero conocer las formas es haber comprendido la unidad de la naturaleza en medio de las materias más desemejantes, y por consiguiente, poder descubrir y producir fenómenos y operaciones hasta aquí desconocidos [...] Así, pues, del descubrimiento de las formas resulta una teoría verdadera y una amplia práctica" ${ }^{\text {. }}$.

Desde esta perspectiva, la sentencia "saber es poder", interpretada típicamente como consigna del dominio del hombre y del conocimiento sobre la naturaleza y sobre sí mismo,

\footnotetext{
${ }^{4}$ Francisco Larroyo. Estudio introductorio a Instauratio Magna. En: Francis Bacon. Instauratio Magna. Buenos Aires, Argentina, 1980. pp. xxiv-xxv.

${ }^{5}$ Pérez-Ramos. Op. cit., p. 140.

${ }^{6}$ Ibídem, p. 141.

${ }^{7}$ Francis Bacon. 1980. Novum Organum. trad. Francisco Larroyo. Buenos Aires, Porrúa. II. xvii. Desde ahora, este texto se citará NO. En este sentido, es emblemática la ley o forma del calor propuesta por Bacon: "resulta que la forma o la definición verdadera del calor puede expresarse así en pocas palabras: El calor es un movimiento expansivo, combatido que obra en las moléculas del cuerpo. Al carácter de la expansión es preciso añadir que éste es u un movimiento del centro a la circunferencia juntamente con un movimiento de abajo a arriba. A este otro carácter del movimiento, acción molecular, es preciso añadir que la acción se verifica sin lentitud, con cierta rapidez y hasta con impetuosidad.

Para la práctica, método conforme a esta definición. Tal es, en efecto, el procedimiento general: si en un cuerpo cualquiera se puede determinar un movimiento de dilatación o de expansión, al propio tiempo comprimir y empujar hacia atrás ese movimiento, de suerte que no tenga un curso tranquilo, sino que proceda a través de alternativas de acción y reacción, indudablemente se producirá calor" $(N O$, II. $\mathrm{xx})$

${ }^{8}$ NO, II. iii
} 
se restringe al campo de la epistemología, en tanto denota un tipo de saber que no busca esencias, sino modos de operar. En efecto, conocer es poder producir el mismo fenómeno ahora en condiciones artificiales.

La conclusión a la que ha llegado Pérez Ramos por vía de la epistemología ha sido compartida por otros autores que han estudiado distintos aspectos de Bacon. Insistiendo en el valor que Bacon otorga a la caridad y la fraternidad, autores como Rose Mary Sargent, Paolo Rossi, John Channing Brings e incluso Reale y Antiseri, han defendido la tesis según la cual el ideal baconiano se inscribe dentro de valores morales extraídos del humanismo y en general del renacimiento que poco tienen que ver con el utilitarismo ${ }^{9}$. En todo caso, su proyecto se inscribe dentro de la reforma del conocimiento que está inspirada en dar al hombre el lugar en la naturaleza que Dios le ha otorgado.

Despojar a Bacon de aquella imagen que parte de la tradición filosófica le ha dado resultados interesantes y fecundos para estudiar la figura del filósofo ante la técnica. En efecto, con ello poco a poco nos despojaremos también de la idea de que la técnica es por ella misma deshumanizante y que, por ende, al filósofo no le queda otra opción sino tomar distancia frente a ella. El otro extremo también debe evitarse: no se trata tampoco de asumir el proyecto tecnológico sin criterio alguno; sigue teniendo vigencia la idea heideggeriana de que se requiere una actitud de serenidad frente a la técnica. Nuestra posición es, más bien, que los nuevos entornos tecnológicos llaman constantemente al filósofo a pensar su quehacer en ellos.

En esta línea -en el asunto de pensar el oficio del filósofo al lado de la tecnología-, Bacon puede servir como referente, al menos como punto de comparación. Al lado de los postulados referidos al método de la filosofía (la inducción), Bacon desarrolló un arduo trabajo para definir lo que Gaukroger llama el "buen sentido y comportamiento del científico" 10 en el contexto del saber operativo. Bien podríamos decir que el filósofo natural, cual es el filósofo al que Bacon se refiere, encarna dos actitudes o valores fundamentalmente: la humildad y la caridad.

Antes de exponer sus tesis sobre la nueva filosofía y sobre el nuevo método, Bacon se propuso la tarea de crítica y refutación de las falsas filosofías. En un texto anterior al Novum Organum, La refutación de las filosofías, Bacon desarrolló esta crítica atendiendo fundamentalmente a las actitudes reprochables de los falsos filósofos. La idea que guió esta

\footnotetext{
${ }^{9}$ Cfr. Sargent, Rose-Mary. "Francis Bacon and the Humanistic Aspects of Modernity". En: Midwest Studies in Philosophy. Volume n..$^{\circ}$ XXVI: Renaissance and Early Modern Philosophy. French, Peter y Wettstein, Howard [edits.]. Oxford, Blackwell, 2002, pp. 124-139; también, de la misma autora, "Bacon as an advocate dor cooperative scientific research". En: Peltonen, Markuu. The Cambridge Companion to Bacon. Cambridge University Press, 1999, pp. 146-171. De Paolo Rossi puede consultarse su conocido libro Francis Bacon: de la magia a la ciencia. Traducción de Susana Gómez López. Madrid, Alianza, 1990. O su artículo "Bacon's idea of science". En: Peltonen, Op. cit., pp. 25-46. De John Channing Brings véase su artículo "Bacon's science and religión". En Peltonen, op. cit., pp. 173-199. De Reale y Antiseri puede consultarse la Historia del pensamiento filosófico y científico. Tomo segundo: "Del Humanismo a Kant". Barcelona, Herder, 1988. Traducción de Juan Andrés Iglesias.pp. 303-304.

${ }^{10}$ Stephen Gaukroger. Francis Bacon and the transformation of the early-modern Philosophy. Cambridge University Press, 2004, p. 12
} 
crítica es propiamente la acusación de soberbia de los filósofos especulativos: "a decir verdad, el obstáculo para el asunto que ahora tratamos no fue tanto su oscuridad o dificultad cuanto la soberbia humana, a cuyos ojos la naturaleza misma está manchada en gran parte, y precisamente en la parte mejor; esta soberbia empuja a los hombres hasta tales extremos de locura que no auscultan el latido de la naturaleza sino su propio latir, y aunque crearan artes para auscultarlo, no darían con él"11.

Bacon presenta a Aristóteles como aquel filósofo que, en lugar de callar y dejar que la naturaleza hablase, impuso su propio pensamiento sobre ella: "comenzando por Aristóteles, ponemos a vuestra memoria por testigo, hijos míos, de si en sus libros físicos y metafísicos no oís más a menudo las voces de la dialéctica que las de la naturaleza"12. Este encierro del conocimiento en la telaraña que se había creado para atrapar la naturaleza constituye una de las críticas más fuertes al aristotelismo y a la escolástica en particular. Los escolásticos se quedaron con el armazón pero carecían de contenido para ampliar su conocimiento, de ahí que se enredaran en vanas discusiones interminables. La escolástica es "una filosofía sin contenidos que crea palabras nuevas y hace que a estas palabras correspondan sustancias"13. La lógica que desarrolla Bacon mantiene el esfuerzo emprendido por Aristóteles por depurar la filosofía de elementos fantásticos, pero se trata de una lógica que permite descubrir formas que, entendidas ya no como esencias sino como maneras de producir los fenómenos, no requieren saltar a la teología, escapan a las telarañas conceptuales y generan operaciones sobre la naturaleza. La arrogancia de Aristóteles, además, señala Bacon, lo llevó a despreciar cualquier filosofía anterior a él: "y si hay alguien en filosofía que viene en nombre propio, ese es Aristóteles, que en todo se tiene a sí mismo por la autoridad, y que de tal modo despreció a los antiguos que casi ni se digna nombrar a ninguno de ellos, como no sea para refutarlo e injuriarlo"14. Platón, por su parte, "no se preocupó gran cosa por la filosofía natural, salvo en la medida indispensable para defender su nombre y su fama de filósofo, así como para añadir e infundir cierta grandeza a sus doctrinas morales y políticas"15.

Puede notarse que la acusación de soberbia está íntimamente ligada con la crítica al método dialéctico, pues este, en lugar de investigar la naturaleza, se dedica a imponer a esta sus categorías y a sacar conclusiones silogísticamente ${ }^{16}$. Precisamente, desde aquí puede entenderse la diferencia que propone en el Novum Organum entre las anticipaciones o prenociones de la naturaleza y su interpretación. En efecto, las primeras son ideas que surgen prematuramente en el hombre y le imponen a la naturaleza elaboraciones rápidas que terminan desdeñándola ${ }^{17}$. La interpretación, por el contrario, parte de la experiencia y

\footnotetext{
${ }^{11}$ Francis Bacon. Refutación de las filosofías. Edición y traducción de J. M. Artola y M. F. Pérez. Madrid, Consejo Superior de Investigaciones Científicas, 1985, p. 115.

12 Ibídem, p. 89.

${ }^{13}$ Paolo Rossi. Francis Bacon: de la magia a la ciencia. Traducción de Susana Gómez López. Madrid, Alianza, 1990, p. 141.

${ }^{14}$ Francis Bacon. Refutación de las Filosofías. Op. cIt., p. 93.

${ }^{15}$ Ibídem, p. 101.

${ }^{16}$ Sobre la crítica de Bacon al silogismo puede verse Gonzalo Serrano. La querella en torno al silogismo 1605-1704: conocimiento versus forma lógica. Bogotá: Universidad Nacional de Colombia, 2006, pp. 33-94. ${ }^{17}$ NO.I.XX
} 
en un espíritu paciente y reflexivo se eleva gradualmente hasta las leyes que son familiares a la naturaleza ${ }^{18}$, en esto consiste la inducción.

Bacon considera que exponer claramente la inducción, contrario a los filósofos que criticó, es plenamente un acto de humildad: "En este sentido, efectivamente, nos felicitamos muy de veras, y no sólo consideramos que somos afortunados, sino también que hemos prestado un buen servicio al género humano, por haber puesto de manifiesto de qué es capaz un verdadero y legítimo acto de humildad del espíritu humano" ${ }^{\text {"19 }}$.

El conocimiento de la naturaleza no es, no obstante, un bien con el que el filósofo deba quedarse. Bacon recurre a la primera carta de San Pablo a los Corintios para explicar el fin del conocimiento: "si yo hablara con las lenguas de los hombres y de los ángeles, y no tuviera caridad, sería como címbalo que resuena ${ }^{20}$; no porque el hablar con las lenguas de los hombres y de los ángeles no sea cosa excelente, sino porque, si se separa de la caridad y no se aplica al bien de los hombres y de la humanidad, es más gloria resonante e indigna que virtud meritoria y sustancial"21; Bacon motiva a que "aspiren los hombres a un avance o progreso ilimitado en ambas (filosofía y teología), cuidando, eso sí, de aplicarlas a la caridad y no al envanecimiento, a la utilidad y no a la ostentación" ${ }^{\text {22 }}$. El provecho, pues, de la filosofía natural, cual es extender el imperio del género humano sobre la naturaleza -que no el imperio de un solo hombre sobre su país o de un país sobre otro- es la ambición más sabia que pueda tener el género humano ${ }^{23}$.

El llamado a la humildad y a la caridad que hace Bacon a los filósofos, unido a la propuesta de una nueva lógica de investigación natural, implica nuevas prácticas para el filósofo; prácticas que intentan darle plomo y peso y no alas al espíritu humano de modo que esté más cerca del mundo natural y no se eleve en especulaciones ${ }^{24}$. Dado que es la anticipación lo que no permite un conocimiento familiar a la naturaleza, el nuevo método intenta que el investigador no involucre su imaginación en el proceso de conocimiento. En este aspecto, Bacon critica las artes, que dependen del genio individual; en lugar de ello, el método debe ser tal que aproxime las inteligencias ${ }^{25}$; que el conocimiento surja de las reglas del método y de las demostraciones y no de la natural excelencia de la naturaleza ${ }^{26}$. En oposición al conocimiento que se guía por la obra de un genio, así como a la tradición filosófica que no ha avanzado sustancialmente luego del aporte de algunos filósofos griegos, la nueva filosofía es obra de muchos, requiere el esfuerzo aunado de los filósofos, a ejemplo de las artes mecánicas.

\footnotetext{
${ }^{18}$ NO.I.XXII

${ }^{19}$ Francis Bacon. Refutación de las Filosofías. Op. cit., p. 155.

${ }^{20} 1$ Cor. 13,1

${ }^{21}$ Francis Bacon. El avance del saber. Introducción de Alberto Elena, traducción y notas de María Luisa Balseiro. Madrid: Alianza, 1988, p. 24.

${ }^{22}$ Ibídem, p. 25.

${ }^{23}$ NO.I.CXXIX

${ }^{24}$ NO.I.CIV

${ }^{25}$ NO.I.LXI

${ }^{26}$ NO.I.CXXII
} 
En la tradición platónico-aristotélica, el desarrollo de la razón supone la valoración de las artes liberales sobre las artes mecánicas. Las primeras son más nobles, pues liberan el espíritu, mientras las segundas atan al mundo del provecho y de la subsistencia. El rechazo de Bacon a esta tradición implica también la inversión en la valoración de las artes, inversión que era corriente en los siglos XVI y XVII ${ }^{27}$. Más aún, las artes mecánicas brindarían el modelo para construir una nueva ciencia y una nueva cultura cuyo propósito fundamental es la trasformación de la naturaleza en beneficio humano ${ }^{28}$.

Las artes mecánicas muestran el modelo científico. El modelo contemplativo, pero más fuertemente el de la magia, sustenta una forma de ser científico según la cual sólo algunos seleccionados, a través de un momento de soledad en el cual son iluminados y reciben conocimiento, son capaces de construir saber. El saber se convertía en un secreto que no podía ser de conocimiento público, pues solo algunos estaban en capacidad de comprenderlo y de responder por él. En franca oposición, las artes mecánicas muestran que la construcción científica se construye en colectivos y por sucesión de conocimiento de generación en generación ${ }^{29}$. Aquí el conocimiento construido con las manos se impone al adquirido por vía de inspiración racional. El desarrollo de un método de conocimiento que funcione casi mecánicamente, es decir, que no dependa de condiciones o características particulares, responde al rechazo del saber hermético-mágico.

\section{La idea de tecnología: el tercer entorno}

Estudiando a Bacon hemos querido mostrar que existe una íntima relación entre las ideas de técnica y filosofía, por un lado, y el oficio del filósofo, por otro. En lo que resta de este escrito intentaremos mostrar esta misma relación teniendo como referente, no ya la técnica "industrial", sino la tecnología de la información y la comunicación. Hemos asumido el concepto de tercer entorno como una manera de caracterizar el desarrollo e impacto que ha tenido la tecnología de la información y la comunicación en lo que Castells llama la era de la información. Ubicados en este concepto intentaremos señalar algunas líneas que moldean el oficio del filósofo.

La idea de tercer entorno recupera y actualiza ideas desarrolladas por Ortega en Meditación de la técnica ${ }^{30}$. Ortega, queriendo responder a la pregunta ¿qué es la técnica?, da un paso más allá frente a la idea de técnica -y de mundo- como máquina, idea que subyace al desarrollo técnico moderno ${ }^{31}$, y la define como sobrenaturaleza. En su empeño por estar en el mundo, señala, el hombre se ve ante una serie de dificultades impuestas por su circunstancia, que le impiden u obstaculizan la satisfacción de sus necesidades; de allí que deba operar una reforma en ella. Operar sobre ella indica modificar lo que ya existe o crear algo completamente nuevo con miras a que el hombre ahorre esfuerzos en la tarea de vivir y pueda dedicar sus esfuerzos al bienestar, a su proyecto vital. A esta reforma la llama

\footnotetext{
${ }^{27}$ Paolo Rossi. De la magia a la ciencia. Op. cit., p. 42.

${ }^{28}$ Ibídem, p. 84.

${ }^{29}$ Ibídem, p. 77.

${ }^{30}$ Ortega y gasset. Meditación de la técnica. Madrid, Revista de Occidente, 1968.

${ }^{31}$ Cfr. Ibídem, p. 53.
} 
Ortega una sobrenaturaleza. Esta es la técnica, "que podemos, desde luego, definir como la reforma que el hombre impone a la naturaleza en vista de la satisfacción de sus necesidades" 32 . La técnica, como reforma, configura una nueva naturaleza que en principio se ubica entre el hombre y la naturaleza; entre él, que tiene en su mira realizar su proyecto vital, y la naturaleza, a la cual encuentra como un complejo de facilidades y dificultades para realizar tal proyecto $^{33}$, complejo que bien puede denominarse naturaleza, mundo o circunstancia $^{34}$. Dicha sobrenaturaleza, una vez instalada en el complejo vital humano, es, a su turno, naturaleza.

En efecto, en este texto de Ortega se comienza a vislumbrar que el desarrollo técnico no sólo debe ser considerado como un útil, sino que empieza a comprenderse como un lugar donde habita el hombre, donde se desenvuelve: como entorno. Las teletecnologías, en especial las redes como Internet, han permitido la canalización y expresión de ideas libertarias, democráticas y antiautoritarias, precisamente por su estructura interactiva. Así, este tipo de tecnologías representan un cambio profundo no sólo en cuanto a desarrollo técnico, sino también, como señala Castells, en cuanto al impacto social y cultural que ellas crean. Por esto, propiamente hablando, hay aquí un cambio de paradigma tecnológico ${ }^{35}$.

Apoyado sobre esta base, Javier Echeverría ha desarrollado la idea de entender la sobrenaturaleza que crea la tecnología telemática como tercer entorno ${ }^{36}$, aspecto que no había sido desarrollado con la técnica moderna. En cuanto entorno, la tecnología se nos presenta como espacio social, como espacio de actuación e interacción; de aquí que el tercer entorno pueda entenderse también bajo la imagen de una ciudad, de una Polis ${ }^{37}$, en la cual, como lo ha señalado Platón, confluyen e interactúan una pluralidad de actividades y oficios humanos requeridos para el mantenimiento de la Polis misma ${ }^{38}$.

Ahora bien, este autor caracteriza el E3 como entorno telemático, y lo contrapone al segundo entorno (E2), aquel que gira en torno al ambiente social de la ciudad, y al primer entorno (E1), el que gira en torno al ambiente natural humano ${ }^{39}$. Si bien el tercer entorno no reemplaza al segundo, así como la aparición del segundo no implicó la desaparición del

\footnotetext{
${ }^{32}$ Ibídem, p. 26.

${ }^{33}$ Ibídem, p. 48.

${ }^{34}$ Ibídem, pp. 48-49

${ }^{35}$ Sobre la idea de cambio de paradigma tecnológico o revolución tecnológica que generan las TIC, véase Manuel Castells. La era de la información. Tomo I. México: Siglo XXI editores, 2006, pp. 61-88.

${ }^{36}$ Javier Echeverría. Los señores del aire: telépolis y el tercer entorno. Barcelona: Destino, 2004.

${ }^{37}$ Ibídem, pp. 156-158. Frente a los conceptos de Telépolis y Cibercultura Echeverría prefiere el de Tercer entorno, dado que este abarca no sólo el nuevo espacio social que se abre con Internet, sino el que se abre con la telemática: teléfono, radio, televisión, dinero electrónico, multimedia, hipertextos y redes telemáticas (dentro de las cuales está Internet) Ibídem, p. 48. Para nosotros, si bien este escrito versa solamente sobre el oficio del filósofo en Internet, el concepto de Tercer entorno resulta interesante, pues evidencia una idea de tecnología distinta a la moderna; asunto que es importante para los propósitos de este escrito.

${ }^{38}$ Precisamente, el libro de Echeverría que aquí estudiamos, en gran parte está dedicado a sustentar que el tercer entorno no es solamente propicio para acciones comunicativas. Esto lo muestra haciendo seguimiento a distintas acciones que se dan efectivamente dentro de él: guerra, violencia, actividades financieras, teletrabajo, ciencia, arquitectura, urbanismo, ocio, educación, medicina, escritura, arte, cultura, periodismo y la idea de cuerpo.

${ }^{39}$ Ibídem, pp. 13-14.
} 
primero, sí afecta las condiciones de interacción en estos dos. Por ejemplo, los estudios de Castells $^{40}$ dan cuenta de ello. No obstante, nos interesa aquí lo que ocurre en el interior del tercer entorno, no su relación con el segundo. La diferencia fundamental entre el tercero y los otros dos, señala Echeverría, estriba en que en el tercero se puede actuar e interactuar ${ }^{41}$ a distancia, simultáneamente o no ${ }^{42}$. La acción a distancia que permite estas tecnologías no se restringe al campo de las acciones comunicativas. Su gran potencial reside, más bien, en que en ellas son posibles las acciones militares, financieras, educativas, lúdicas, etc.

Dada la idea de tecnología como tercer entorno, cabe preguntarnos por la acción del filósofo allí; en qué consiste la labor filosófica en esta polis, como parte de una pluralidad de oficios; qué querría decir realizar acciones filosóficas en este entorno. Ese es, propiamente, nuestro campo de estudio. Vale la pena apuntar que concordamos con Javier Echeverría en que estas y otras preguntas de este estilo son parte de la tarea de humanizar el tercer entorno, en el sentido en que este debe propiciar la vida humana dentro de él. Retomando la terminología orteguiana, debemos decir que el tercer entorno debe posibilitar la realización del proyecto vital de cada ser humano que pertenezca a él.

\section{El oficio del filósofo en el tercer entorno}

En aras de especificar nuestra pregunta, dedicaremos algunas líneas al tipo de argumento que nos permite adentrarnos en ella. En una primera etapa, se trata de proceder analógicamente. Según esto, así como preguntamos por el papel del filósofo en la vida del segundo entorno, o frente a la técnica industrial, preguntamos por el papel del filósofo en la vida del tercer entorno, frente a las tecnologías de información y comunicación.

La pregunta analógica es fecunda en este caso, pues permite crear una idea de algo desconocido (tema), a partir de similitudes que esto tiene con otra cosa (foro). Así, la analogía permite explicar, estructurar o aclarar el tema en virtud de lo que se sabe del foro $^{43}$. En nuestro caso, la analogía permite conocer la labor filosófica en el tercer entorno (tema de la analogía), a partir de lo que sabemos de ello en el segundo entorno (foro). Entre uno y otro domino, en efecto, se han establecido rasgos similares que nos permiten plantear la relación analógica: por ejemplo, la idea de que los tres ámbitos son entendidos como entornos, la idea de polis, la idea de acción e interacción en los entornos e incluso la idea de

\footnotetext{
${ }^{40}$ Véase por ejemplo Manuel Castells. La era de la información. Tomo I, op. cit.

${ }^{41}$ Es importante destacar la distinción entre actuar e interactuar. En efecto, con la aparición de algunas tecnologías como el teléfono, las redes telemáticas, los hipermedios e hipertextos, se da un paso en cuanto a la actuación del usuario. En tecnologías como el televisor y el radio, los usuarios son pasivos, mientras que en estas otras, los usuarios son activos; de allí que se hable de interacción. Esto marca una gran diferencia, por ejemplo, en el caso de la actitud frente a la tecnología. En el primer caso nos encontramos con críticas a la técnica, como las hiciera la Escuela de Frankfurt, mientras que en el segundo, con el realce de la tecnología como factor fundamental dentro de la consolidación de proyectos comunitarios, como lo explica Vattimo en Una sociedad transparente. (Barcelona, Paidós-I.C.E.-U.A.B., 1998, pp. 73-89).

${ }^{42}$ En este aspecto radica la divergencia de la propuesta de Echeverría frente a la de Castells en lo que se refiere a caracterizar la acción social. Para Castells, la acción social se da siempre en simultaneidad; Echeverría propone que la simultaneidad no es requisito para la acción social. Cfr. Ibídem, pp.148-154.

${ }^{43}$ Chäim Perelman. El imperio retórico. (A. L. Giraldo, Trad.) Bogotá: Norma, 2004, p. 154.
} 
que la filosofía puede hacer presencia en ellos ${ }^{44}$. Pero entre ellos hay también diferencias, asunto por el cual no planteamos una relación de identidad sino de analogicidad. Algunas de ellas pueden ser el sustrato material de cada uno de los entornos, los canales comunicativos, las nociones de tiempo y espacio, etc. ${ }^{45}$.

El estudio de Bacon se dirige en esta dirección: ha mostrado un camino en el cual el filósofo actúa del lado de la tecnología. Constatar este hecho permite pensar que el filósofo también podría actuar dentro del tercer entorno, al lado de la tecnología. Que su actitud frente a ella no siempre es de rechazo o de crítica, que podría ser también de construcción. No obstante, no se espera que se plantee su oficio en los mismos términos que lo ha hecho Bacon, dado que no nos encontramos frente a la misma idea de filosofía y de técnica. En efecto, no proponemos la labor del filósofo como filósofo natural, más bien, nos movemos en la idea de la filosofía como comprensión, como argumentación, incluso como crítica y liberación; aspectos que se inscriben directamente con el giro lingüístico y que nos obligan a pensar la labor de filósofo del lado de la construcción de sociedades democráticas e igualitarias. Como vimos, tampoco se propone la técnica de la misma manera en que se hacía en el siglo XVII.

Es importante señalar que, si bien la analogía se establece en virtud de similitudes, las diferencias deben ser anunciadas para comprender los asuntos que se relacionan. En efecto, el camino de la analogía sirve para consolidar una primera idea acerca del trabajo del filósofo en la técnica, construida a partir de ideas anteriores similares. No obstante, debemos preguntar también por las condiciones propias, o nuevas, que tienen estos entornos para la labor filosófica, por aquello que lo diferencia.

¿Qué elementos podríamos extraer acerca de la labor del filósofo que hemos heredado y qué elementos surgen actualmente frente a las tecnologías de la información y comunicación? Habiendo indicado una idea de técnica, nos resta señalar algunas perspectivas que pueden contribuir a este propósito. Creemos que al dar respuesta a estas preguntas a la vez respondemos aquella que planteábamos más arriba acerca de cómo humanizar el tercer entorno: desarrollando la labor propia del filósofo dentro de él.

1. El filósofo ante la información. La tecnología actual ha permitido el flujo de enormes volúmenes de información a lo largo de la red; la posibilidad de conexión con otras fuentes de consulta, aparte de las que se encontraban circunscritas al ámbito local, es ahora una realidad. Mientras que en el segundo entorno el investigador en filosofía tenía por referente principalmente la bibliografía que circulaba en las bibliotecas cercanas a su lugar de residencia, en el tercer entorno tiene la posibilidad de acceder a textos disponibles en otros lugares. Así las cosas, el filósofo se ve ante el reto de optimizar los procesos de búsqueda, organización y procesamiento de información.

\footnotetext{
${ }^{44}$ De hecho, como el mismo Echeverría lo señala en su libro citado, es importante reconocer las analogías entre los tres entornos. Como ejemplo de esto, piénsese en la analogía ente el escritorio del segundo y el tercer entorno, que ha dado lugar a que se construyera la interfaz y funciones del sistema operativo de Windows.

${ }^{45}$ Echeverría señala veinte "propiedades diferenciales" que permiten caracterizar el tercer entorno; estas características se elevan por comparación con el segundo entorno. Cfr. Op. Cit., pp. 57-146.
} 
Mientras Bacon centraba el oficio del filósofo en torno a la investigación de la naturaleza, la técnica actual llama a trabajar en el procesamiento de información: apoyado en herramientas computacionales, el filósofo organiza y recoge datos, textos y bibliografías; estudia, analiza y refuta ideas y argumentos; escribe y publica sus investigaciones. Con ésto no queremos decir que en el segundo entorno el manejo de información no fuera preocupación de los filósofos (de hecho, una de las preocupaciones de Bacon era precisamente que el filósofo debía escribir sus resultados, sistematizarlos, publicarlos); en lugar de ello, queremos resaltar que este tópico ha cobrado gran importancia hoy y que gran parte del trabajo consiste en procesarla; de hecho, bajo este propósito ha avanzado gran parte de la inteligencia artificial.

2. El filósofo y las máquinas inteligentes. Uno de los grandes retos de la segunda mitad del siglo XX ha sido el desarrollo de la inteligencia artificial, no solo por lo que implica su diseño, sino por las preguntas e impactos sociales que genera. Mientras Bacon quería que la labor del filósofo pudiera utilizarse en el diseño de artefactos con miras a verificar la verdad del conocimiento natural, hoy el diseño de máquinas inteligentes las convierte en piezas claves para la comprensión de las estructuras del pensamiento y la subjetividad, por ejemplo en los planteamientos de la epistemología experimental dentro del campo de la fenomenología.

Dentro de la amplia gama de posibilidades que esto abre, nos interesa mostrar que, gracias a estos desarrollos, hoy el filósofo puede argumentar con agentes no humanos. Mientras que la tarea de "pensar" era atribuible solo a seres humanos, y para algunos también a Dios, ahora algunas de las características de la "cosa que piensa" podemos atribuirlas también a agentes computacionales. En la medida en que este campo avance, podemos prever sus consecuencias: no seremos los únicos agentes inteligentes en la construcción de argumentos, en las discusiones, y quizás en la vida civil del tercer entorno. Mientras Bacon pretendía que el hombre alcanzara el dominio del mundo natural con el apoyo de máquinas, ahora nos corresponde construir vida humana en medio de la interacción con máquinas que piensan como nosotros.

3. El filósofo y la interacción. El mundo que proponía Bacon no incluía hombres y máquinas interactuando en cuanto seres pensantes; las máquinas ayudarían en el desarrollo y bienestar humanos en el desarrollo de tareas, en la simulación de procesos naturales y en el descubrimiento de la verdad, como se narra en la Nueva Atlántida. En el tercer entorno, la interacción amplía sus rutas. Por un lado, la interacción con las máquinas no se hace sólo con fines útiles. No sorprende decir que la construcción del tercer entorno, su civilización y humanización, es una tarea de humanos y máquinas interactuando. Esto lo vemos hoy en chatbots como A.L.I.C.E. ${ }^{46}$, entre otros ejemplos, y suponemos que cada día esta interacción se irá perfeccionando, en la medida en que se tienda a superar la prueba de Turing.

\footnotetext{
${ }^{46}$ Véase en: http://alice.pandorabots.com/ Fecha de consulta: agosto de 2008
} 
De otro lado, la interacción con otros humanos hoy es posible hacerla a distancia. De nuestro mundo de la vida hoy hacen parte personas que no están en nuestro entorno corporal ni local. Así, la conformación de comunidades se ha intensificado y fortalecido, con lo cual el trabajo filosófico se realiza en conjunción o con el apoyo de gran cantidad de pares que desde distintos contextos y perspectivas evalúan y ejercen crítica sobre los productos: el trabajo filosófico se ha vuelto trabajo colaborativo.

4. El filósofo y el trabajo colaborativo. Los volúmenes de información, la presencia de máquinas pensantes y la posibilidad de interacción con comunidades globales confluyen en la idea del trabajo filosófico como trabajo colaborativo. Más que a la imagen del filósofo actuando solo, apartado de la vida civil o sumergido en reflexiones aisladas, la técnica actual llama a un filósofo conectado con los demás, actuando dentro de equipos de trabajo, reflexionando y discutiendo con sus pares, preocupado por asuntos de interés compartido con otros.

Quizás este sea uno de los aspectos en los que más aportaría Bacon en la configuración actual del oficio del filósofo. Bacon comprendió que hacer filosofía era tarea de equipos de trabajo, incluso, que el proyecto de conocer y dominar la naturaleza pasaba de generación en generación y no debía ser fruto del genio de unos pocos hombres y de la repetición de los demás. Del mismo modo, civilizar el tercer entorno, y aun el segundo, es tarea de muchos, no de unos pocos, es construcción colectiva, compete a las comunidades que allí interactúan.

\section{Consideraciones finales}

Si bien hemos defendido la idea de que el oficio del filósofo hoy está íntimamente ligado a la aparición de las tecnologías de la información y la comunicación, las cuatro líneas que acabamos de indicar deben ser acotadas a partir de los riesgos que corren paralelos a ellas. Quizás sea pertinente recordar no solo lo que el filósofo puede hacer al lado de la técnica, sino también la distancia que en ocasiones debe tomar, de modo que su cercanía no le provoque el efecto contrario: la deshumanización.

1. El trabajo de procesamiento de la información podría llevar a lo que Bacon quiso evadir: la repetición de las ideas de unos pocos y el consecuente estancamiento de la filosofía. A la par de dicho procesamiento, se requiere la generación de ideas nuevas, de nuevas perspectivas y problemas que garanticen el avance del saber. La generación de pensamiento debe evitar también que se caiga en una recepción acrítica de la tecnología, como se decía que podía ocurrir con la recepción de la radio y la televisión.

2. El miedo ante un dominio de las máquinas sobre el hombre, un temor muy difundido sobre todo en los medios de comunicación y en el cine; o el temor a que por imitación de la racionalidad de las máquinas el hombre se limite a la racionalidad instrumental, ha sido una constante en el pensamiento sobre técnica en el siglo $\mathrm{XX}^{47}$. Si bien podría objetarse que muchas de estas ideas carecen de fundamento, o que pueden ser vistas como críticas a la idea de técnica como máquina, más no a la de técnica como tercer entorno, no se debe descuidar el hecho de que el desarrollo técnico es solo un medio para

\footnotetext{
${ }^{47}$ Temores representados principalmente por la Escuela de Frankfurt.
} 
alcanzar los fines humanos. En términos orteguianos, cabe recordar entonces la idea de entorno como sobrenaturaleza que se alza para que el hombre realice su programa vital.

3. Si bien la interacción a distancia ha sido de gran provecho para la ampliación y conexión del mundo, la advertencia que hiciera Mounier en El miedo del siglo XX debe ser revisada. En dicho texto, Mounier denunciaba que actuar a distancia puede desembocar en el olvido del otro. En efecto, si el otro no está presente, puede ocurrir que me olvide de él. El efecto producido sería el contrario al propuesto: en lugar de acercar el mundo, lo estaríamos anulando; de hecho, señala el autor, bajo esas condiciones es posible destruir pueblos enteros con sólo oprimir un botón, como ocurrió con la explosión de las bombas atómicas $^{48}$. Más que una acusación tendiente a anular el uso de la tecnología, se trata de un anuncio que vela por que el ser humano no quede reducido a lo que aparece de él en la pantalla. Se trata, en suma, de considerar su aspecto personal y no sólo su aspecto funcional $^{49}$.

4. Finalmente, el trabajo colaborativo podría llevar al deterioro del trabajo individual, de la reflexión personal, del silencio. Siguen siendo estos aspectos importantes también dentro de la formación y el oficio del filósofo; no obstante, su rol no se agota allí, es sólo un paso dentro del ejercicio de interactuar y relacionarse con otros agentes en la construcción de objetivos comunes.

\section{BIBLIOGRAFÍA}

BACON, Francis. El avance del saber. Introducción de Alberto Elena, traducción y notas de María Luisa Balseiro. Madrid: Alianza, 1988.

Novum Organum. [trad.] Francisco Larroyo. Buenos Aires: Porrúa, 1980.

Refutación de las filosofías. Edición y traducción de J. M. Artola y M. F. Pérez. Madrid: Consejo Superior de Investigaciones Científicas, 1985.

CAStells, Manuel. La era de la información. Tomos I, II y III. México: Siglo XXI Editores, 2006.

Channing Brings, John. "Bacon's science and religión". En Peltonen, Markuu. The Cambridge Companion to Bacon. Cambridge University Press, 1999, pp. 173-199.

ECHEVERRÍA, Javier. Los señores del aire: telépolis y el tercer entorno. Barcelona: Destino, 2004.

LARROYO, Francisco. Estudio introductorio a Instauratio Magna. [aut. libro] Francis Bacon. Instauratio Magna. Buenos Aires, Argentina, 1980.

GAUKROGER, Stephen. Francis Bacon and the transformation of the early-modern Philosophy. Cambridge University Press, 2004.

MOUNIER, Emmanuel. El miedo del siglo XX. Madrid: Taurus, 1957.

ORTEGA y GASSET, J. Meditación de la técnica. Madrid: Revista de Occidente, 1968.

Perelman, Chäim. El imperio retórico. Traducción de A. L. Giraldo. Bogotá: Norma, 2004.

PÉREZ-RAmos, Antonio. Francis Bacon's idea of science: and the Maker's Knowledge Tradition. Oxford: Clarendon Press, 1988.

\footnotetext{
${ }^{48}$ Cfr. Emmanuel Mounier. El miedo del siglo XX. Madrid: Taurus, 1957.

${ }^{49}$ Sobre este tema particular puede consultarse Prada, Maximiliano. "El miedo a la tecnología en la educación: entre la posibilidad y el límite”. En: Revista Pedagogía y Saberes. Bogotá, v. 26, pp. 67-74, 2007.
} 
PRADA, Maximiliano. "El miedo a la tecnología en la educación: entre la posibilidad y el límite”. Revista Pedagogía y Saberes. Bogotá, v. 26, pp.67-74, 2007.

REALE, Giovanni y ANTISERI, Darío. Historia del pensamiento filosófico y científico. Tomo segundo: del Humanismo a Kant. Barcelona, Herder, 1988. Traducción de Juan Andrés Iglesias.

Rossi, Paolo. Francis Bacon: de la magia a la ciencia. Traducción de Susana Gómez López. Madrid: Alianza, 1990.

"Bacon's idea of science". In: Peltonen, Markuu. The Cambridge Companion to Bacon. Cambridge University Press, 1999, pp. 25-46.

SARGENT, Rose-Mary. "Francis Bacon and the Humanistic Aspects of Modernity". In: Midwest Studies in Philosophy. Volume XXVI: Renaissance and Early Modern Philosophy. French, Peter y Wettstein, Howard [edits.]. Oxford, Blackwell, 2002, pp. 124-139.

"Bacon as an advocate or cooperative scientific research". In: Peltonen, Markuu: The Cambridge Companion to Bacon. Cambridge University Press, 1999, pp. 146171.

SERRANO, Gonzalo. La querella en torno al silogismo 1605-1704: conocimiento versus forma lógica. Bogotá: Universidad Nacional de Colombia, 2006.

VAttimo, Gianni. Una sociedad transparente. Traducción de Teresa Oñate. Barcelona: Paidós-ICE-UAB, 1998. 